\title{
Local Swift-BAT active galactic nuclei prefer circumnuclear star formation ${ }^{\star}$
}

\author{
D. Lutz ${ }^{1}$, T. Shimizu ${ }^{1}$, R. I. Davies ${ }^{1}$, R. Herrera-Camus ${ }^{1}$, E. Sturm ${ }^{1}$, L. J. Tacconi ${ }^{1}$, and S. Veilleux ${ }^{2}$ \\ 1 Max-Planck-Institut für extraterrestrische Physik, Giessenbachstraße, 85748 Garching, Germany \\ e-mail: lutz@mpe.mpg.de \\ 2 Department of Astronomy, University of Maryland, College Park, MD 20742, USA
}

Received 23 June 2017 / Accepted 1 September 2017

\section{ABSTRACT}

\begin{abstract}
We use Herschel data to analyze the size of the far-infrared $70 \mu \mathrm{m}$ emission for $z<0.06$ local samples of 277 hosts of Swift-BAT selected active galactic nuclei (AGN), and 515 comparison galaxies that are not detected by BAT. For modest far-infrared luminosities $8.5<\log \left(L_{\mathrm{FIR}}\left[L_{\odot}\right]\right)<10.5$, we find large scatter of half light radii $R_{\mathrm{e}, 70}$ for both populations, but a typical $R_{\mathrm{e}, 70} \lesssim 1 \mathrm{kpc}$ for the BAT hosts that is only half that of comparison galaxies of same far-infrared luminosity. The result mostly reflects a more compact distribution of star formation (and hence gas) in the AGN hosts, but compact AGN heated dust may contribute in some extremely AGN dominated systems. Our findings are in support of an AGN-host coevolution where accretion onto the central black hole and star formation are fed from the same gas reservoir, with more efficient black hole feeding if that reservoir is more concentrated. The significant scatter in the far-infrared sizes emphasizes that we are mostly probing spatial scales much larger than those of actual accretion, and that rapid accretion variations can smear the distinction between the AGN and comparison categories. Large samples are hence needed to detect structural differences that favor feeding of the black hole. No size difference between AGN host and comparison galaxies is observed at higher far-infrared luminosities $\log \left(L_{\mathrm{FIR}}\left[L_{\odot}\right]\right)>10.5$ (star formation rates $\left.\gtrsim 6 M_{\odot} \mathrm{yr}^{-1}\right)$, possibly because these are typically reached in more compact regions.
\end{abstract}

Key words. galaxies: active - galaxies: structure

\section{Introduction}

The evolution of galaxies and their central black holes is linked by the gas supply that is needed for feeding star formation and for accretion onto the black hole, and by feedback effects that the black hole exerts on the galaxy during its phases of activity. There are several related reasons why the "feeding" link between star formation and black hole accretion cannot be tight and why it must be difficult to determine from individual objects or small samples. First, the two phenomena occur on very different spatial scales. Stars may form in gas present on a wide variety of scales at distances from the nucleus between parsecs and $10 \mathrm{kpc}$ or more. In contrast, accretion onto the black hole directly relates only to the material in its immediate surroundings, from the black hole sphere of influence down to the event horizon. Second, and related to the smaller scales, black hole accretion rates (BHAR) and active galactic nucleus (AGN) luminosities can vary rapidly on timescales of years, much shorter than the millions of years or longer for variations of the star formation rates (SFR). As a consequence, the relation between average SFR and BHAR that is observed for large SFR selected samples (e.g., Chen et al. 2013; Delvecchio et al. 2015) is largely erased by the AGN variations when looking at the SFR of samples selected by the instantaneous BHAR (e.g., Shao et al. 2010; Mullaney et al. 2012; Rosario et al. 2012; Stanley et al. 2015; Shimizu et al. 2017).

* Full Table A.1 is only available at the CDS via anonymous ftp to cdsarc.u-strasbg. fr (130.79.128.5) or via

http://cdsarc.u-strasbg.fr/viz-bin/qcat?J/A+A/609/A9
A direct approach to the feeding problem would be to map and compare the molecular gas distributions in active and inactive galaxies, from global gas content down to the smallest scales accessible to current $\mathrm{mm}$ interferometers. This is an expensive project if large samples are needed to probe whether intriguing phenomena in the gas distribution and kinematics (e.g., García-Burillo et al. 2003, 2005; Haan et al. 2009) are indeed more prevalent in active galaxies and related to AGN feeding. Alternatively, star formation can be mapped in active and inactive galaxies using a star formation indicator that is little disturbed by the AGN. In this type of study, Diamond-Stanic \& Rieke (2012) analyzed 84 Seyferts and used circumnuclear star formation rates from mid-infrared polycyclic aromatic hydrocarbon (PAH) features and global star formation rates from extended mid-infrared continuum to argue for a stronger link between BHAR and circumnuclear or kpc scale star formation than between BHAR and global SFR.

The best contrast between the spectral energy distribution (SED) of a star forming galaxy and an AGN SED is reached in the far-infrared, making the far-infrared emission a good tool for measuring SFRs of AGN hosts, except for the AGN with the most extreme ratio of $L_{\mathrm{bol}, \mathrm{AGN}}$ and SFR, for which the contribution of AGN heated dust to the far-infrared can be significant. The PACS instrument (Poglitsch et al. 2010) on board Herschel ${ }^{1}$ (Pilbratt et al. 2010) has dramatically improved sensitivity and spatial resolution of far-infrared photometric

\footnotetext{
1 Herschel is an ESA space observatory with science instruments provided by European-led Principal Investigator consortia and with important participation from NASA.
} 
mapping. Several studies have already used Herschel to study the far-infrared structure of local AGN hosts. Mushotzky et al. (2014) reported a large fraction of almost point like sources ( $\sim 1 / 3$ unresolved sources at $70 \mu \mathrm{m}$, with a 5.8" PSF), small far-infrared (FIR) sizes, and high surface brightnesses in a large $z<0.05$ Swift-BAT selected AGN sample, but with very limited comparison to inactive galaxies. García-González et al. (2016) discuss Herschel images of 33 nearby RSA Seyferts, with nuclei unresolved at FWHM approximately a kpc in $1 / 3$ of the $70 \mu \mathrm{m}$ images and most flux emerging within a radius of $2 \mathrm{kpc}$ for $85 \%$ of the galaxies. Lutz et al. (2016) find far-infrared sizes of a sample of local PG QSOs to be consistent with non-active galaxies of the same FIR luminosity, but the modest sample size and significant fraction of upper size limits, due to the large distance of the QSOs, are limiting factors. Here, we use the Herschel archive to assemble large samples of local AGN and inactive comparisons, and measure and compare in a consistent manner the far-infrared sizes of AGN hosts and other galaxies.

Section 2 discusses the sample and data analysis, Sect. 3 reports the results, and Sect. 4 discusses the size differences we find in terms of the properties of star formation in AGN hosts. We adopt an $\Omega_{\mathrm{m}}=0.3, \Omega_{\Lambda}=0.7$, and $H_{0}=70 \mathrm{~km} \mathrm{~s}^{-1} \mathrm{Mpc}^{-1}$ cosmology, redshift-independent distances from the NASA/IPAC Extragalactic Database (NED), if available, for $z<0.01$ galaxies, a Chabrier (2003) IMF, a conversion $S F R=1.9 \times 10^{-10} L_{F I R=40-120} \mu \mathrm{m}$ as appropriate for the Kennicutt (1998) conversion corrected to Chabrier IMF, and a ratio 1.9 of $8-1000 \mu \mathrm{m}$ IR and $40-120 \mu \mathrm{m}$ FIR luminosity.

\section{Data}

The AGN sample used in this study is based on the 58-month version of the Swift Burst Alert Telescope (BAT) AGN sam$\mathrm{ple}^{2}$ (see also Baumgartner et al. 2013). Uniform sky coverage and selection in 14-195 keV very hard X-rays, which detect all but the fully Compton-thick AGN, make this sample an excellent basis for studies of local moderate luminosity AGN (typical $\left.\log \left(L_{\mathrm{bol}, \mathrm{AGN}}\left[\mathrm{erg} \mathrm{s}^{-1}\right]\right) \sim 44.5\right)$ and their hosts. The $z<$ 0.05 objects of this sample have been observed with HerschelPACS and SPIRE (see Mushotzky et al. 2014; Meléndez et al. 2014; Shimizu et al. 2015, 2016, 2017, for selection, observations, and results from these data). Most were observed in project OT1_rmushotz_1 and the rest in a variety of other projects. We use BAT counterpart identification and X-ray luminosities from these references. When discussing AGN bolometric luminosities, we adopt $L_{\mathrm{Bol}, \mathrm{AGN}}=10.5 \times L_{14-195 \mathrm{keV}}$ (Meléndez et al. 2014).

As non-AGN comparison objects we use a broad set of Herschel-PACS observations of local galaxies. These include the luminous infrared galaxies from RBGS (Sanders et al. 2003) and the KINGFISH galaxies (Kennicutt et al. 2011) that were already used by Lutz et al. (2016) to study local scaling relations of FIR size and surface brightness. In order to improve the number of non-BAT comparison targets in the relevant range of infrared luminosities and redshifts, we searched the Herschel archive for other approved programs with PACS photometric observations of nearby galaxies and retrieved and processed the observations actually obtained. We also included galaxies serendipitously observed in the maps as long as they were clearly identified, were separated from the original target, and had a known redshift. Comparison galaxies may host low luminosity AGN that are below the BAT detection threshold. We did not

2 https://swift.gsfc.nasa.gov/results/bs58mon/ attempt to identify these by other means. Classification as BATAGN or comparison depends solely on detection in the 58-month BAT catalog and not on Herschel observing program, i.e., some of the BAT sources were observed by projects mostly contributing to the comparison sample.

We restricted the BAT-detected sample and the comparison sample to $z<0.06$. This excludes a few higher redshift BAT detected AGN and QSOs observed in other projects, as well as higher redshift comparison galaxies. The redshift cut maintains the systematic BAT redshift selection used by the above references and provides comparison galaxies with similar redshift and scale, avoiding distant and faint targets for which size information is more difficult to obtain given the Herschel beam.

Active galactic nuclei are hosted by massive galaxies (see, e.g., Kauffmann et al. 2003; and, specifically for BAT AGN, Koss et al. 2011). We hence require for our comparison galaxies a minimum near-infrared luminosity as a proxy to stellar mass. For sensitivity reasons, we extract this from the $3.4 \mu \mathrm{m}$ band 1 ALLWISE catalogs that are based on the WISE mission (Wright et al. 2010), and in a few cases our own aperture photometry on WISE $3.4 \mu \mathrm{m}$ images. Specifically, we require $\log \left(v L_{v}(3.4)\left[L_{\odot}\right]\right)>8.5$, which is equivalent to $\log \left(M_{*}\left[M_{\odot}\right]\right) \gtrsim$ 9.5 (e.g., Wen et al. 2013), in good agreement with the total mass range of AGN hosts in Kauffmann et al. (2003) and Koss et al. (2011). Indeed, only one of the BAT AGN hosts discussed below (2MASXi J1802473-145454, $\left.\log \left(v L_{v}(3.4)\right)=8.36\right)$ falls slightly below this threshold, which may also relate to the lack of a redshift-independent distance for this nearby source. For the comparison galaxies that already exclude the powerful BAT AGN, $3.4 \mu \mathrm{m}$ contamination by AGN hot dust is not significant, and we have verified that a cut using 0.5 dex higher $K$-band luminosity from the 2MASS large galaxy atlas, extended source catalog, and point source catalog results in a sample that is very similar to the WISE cut.

Reduction of the PACS data uses the methods described in Lutz et al. (2016). Briefly, we use Herschel archive data processed with SPG 13.0.0; this includes the improved gyroreconstructed pointing history that minimizes effects of pointing jitter on the PSF. For objects that are unresolved or just resolved by Herschel-PACS we use our own dedicated processing, which provides a stable PSF (verified with observations of reference stars), at the expense of not preserving largescale emission. For larger sources we hence use the pipeline JSCANAM (Graciá-Carpio et al. 2015) maps. In both cases, we fit sources detected at signal-to-noise $(\mathrm{S} / \mathrm{N})>10$ with a twodimensional Gaussian, and derive the source FWHM by subtracting the circularized PSF FWHM that is appropriate for the source's far-infrared color from the circularized observed FWHM in quadrature. Weaker or undetected sources are not used for our size analysis. This approach can homogeneously assign a single scale to large samples of Herschel observed targets, in the very highest $\mathrm{S} / \mathrm{N}$ cases down to $1 / 5$ of the PSF. Conversely, as discussed in Lutz et al. (2016), the approach provides only a simplifying measure of the complex structure of nearby galaxies. For example, in the case of a compact circumnuclear starburst superposed on a large disk the fit result may reflect the compact component or the disk, depending on their relative fluxes. We re-use the Lutz et al. (2016) results with the exception of some updated redshift-independent distances and the use of different error maps for fits to KINGFISH galaxies. While we obtained measurements for all three PACS filters if available, we only use in the following the half light radii $R_{\mathrm{e}, 70}$ measured at $70 \mu \mathrm{m}$, because of the sharpest PSF and closer link to active star formation than for longer wavelengths. 

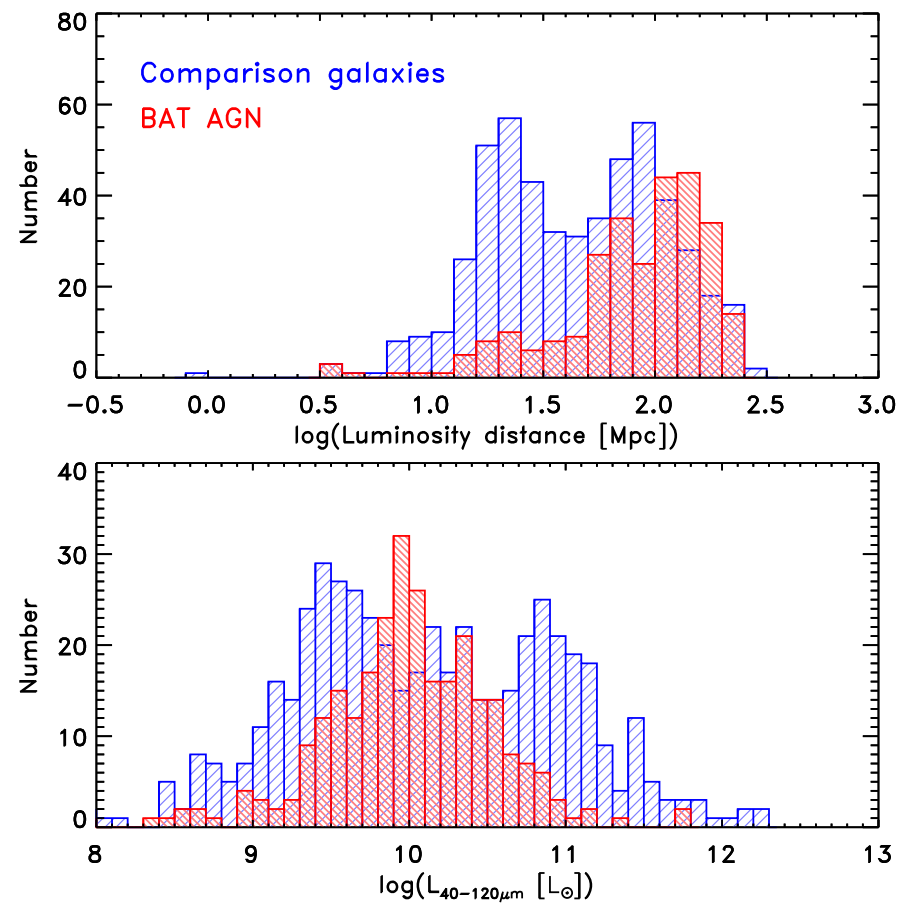

Fig. 1. Distribution of distances and far-infrared luminosities for the samples of $z<0.06$ BAT AGN and non-BAT comparison galaxies.

Most PACS observations of nearby galaxies have used the combination of the 70 and $160 \mu \mathrm{m}$ PACS bands or all three bands including $100 \mu \mathrm{m}$. A notable exception is the Herschel Reference Survey (HRS; Boselli et al. 2010), which used only 100 and $160 \mu \mathrm{m}$ for its PACS observations (Cortese et al. 2014). In order to preserve the many targets of this program and a few others for the non-BAT comparison sample, we scaled the $R_{\mathrm{e}, 100}$ measured from their data to $R_{\mathrm{e}, 70}$ via multiplication by 0.85 , which is the median for non-BAT objects observed in all bands. We have verified that exclusion of these objects, while reducing the comparison object statistics at low infrared luminosities, preserves the immediate result that is reported below in Fig. 2.

Our measurements are not meaningful and accurate for pairs or interacting systems that have a separation of the components on the order of the PSF width, and down to the scales accessible to the method. The measurement then mostly reflects distance and relative strength of FIR emission of the two galaxies rather than the structure of a galaxy. As described in Lutz et al. (2016), we did not fit intermediate separation doubles that would need more complex reduction and fitting schemes, and flagged fit results for closer doubles as identified through multiwavelength data. Neither of the two are used below. BAT AGN and comparison samples are restricted to single galaxies or components of wide doubles that can be analyzed separately.

With these restrictions to $z<0.06$ and to single or widely separated objects, our Herschel archival samples of BAT AGN and comparison galaxies include 277 and 515 galaxies, respectively $^{3}$. Figure 1 shows their distributions in distance and

\footnotetext{
3 Herschel projects contributing to the BAT and/or comparison samples via original targets or serendipitously observed galaxies include KPGT_cwilso01_1, KPGT_esturm_1, KPGT_smadde01_1, KPOT_jdavie01_1, KPOT_rkennicu_1, GT1_ lspinog1_2, GT1_mbaes_1, GT1_msanchez_2, OT1_bholwerd_1, OT1_dsanders_1, OT1_lcortese_1, OT1_lho_1, OT1_rmushotz_1, OT1_sveilleu_1, OT1_vwild_1, OT2_aalonsoh_2, OT2_aleroy_2, OT2_bholwerd_3, OT2_datlee_1, OT2_dpisano_1, OT2_emurph01_3,
}

far-infrared luminosity. The comparison sample covers the range of the BAT AGN. As expected for a purely archival sample, its detailed distribution differs from the BAT detected sample, partly due to the significant numbers contributed by dedicated projects aiming, for example, at typically distant IR luminous galaxies or at nearby (often Virgo cluster) galaxies. We have tested the effect of randomly eliminating $80 \%$ of the $\log \left(D_{\mathrm{L}}[\mathrm{Mpc}]\right)<1.7 \mathrm{com}-$ parison galaxies, a cut that makes the BAT and comparison redshift histograms quite similar. While statistical errors increase, the basic result of Fig. 2 below is again preserved. The difference in $L_{\text {FIR }}$ distributions (Fig. 1 bottom) is considered by our analysis because comparisons are done as a function of $L_{\mathrm{FIR}}$.

Derived half light radii at $70 \mu \mathrm{m} R_{\mathrm{e}, 70}$ for our samples as well as far-infrared and BAT luminosities are listed in Table A.1. Part of the data are shown for guidance; the full table is available at the CDS.

\section{Results}

Half light radii at $70 \mu \mathrm{m}, R_{\mathrm{e}, 70}$ are shown in Fig. 2 as a function of FIR luminosity for both the $z<0.06$ BAT AGN hosts and the $z<0.06$ comparison galaxies. First, as already emphasized by Lutz et al. (2016), there is a large size spread ( 2 dex) of galaxies at moderate IR luminosities; these luminosities can be produced in compact circumnuclear regions and by star formation spread over large disks. Figure 2 shows that such a large size spread is observed for both the BAT AGN hosts and the comparison galaxies, and that the distributions overlap; there is an indication of smaller sizes for the BAT hosts.

In order to quantify this size difference, we have computed the median far-infrared half light radius for both categories and for six bins of 0.5 dex width in logarithm of the far-infrared luminosity. We derive the median value, median absolute deviation MAD, and uncertainty $\sigma=1.4826 \times M A D / \sqrt{N}$, for the half light radius $R_{\mathrm{e}, 70}$ of the objects in each bin. These are plotted in the right panel of Fig. 2 and are listed in Table 1. Given the large scatter of individual objects, the subsample size $N$ in each bin is essential for a meaningful comparison. In the computation of the median and MAD, we have used the upper size limits $(12 \%$ of the BAT hosts and 3\% of the comparisons) at their nominal values, which conservatively underestimates the differences between BAT hosts and comparison galaxies as reported below.

For the modest FIR luminosity bins up to $\log \left(L_{\text {FIR }}\right.$ $\left.\left[L_{\odot}\right]\right)=10.5$, which is $S F R \lesssim 6 M_{\odot} \mathrm{yr}^{-1}$ if the far-infrared is due to star formation, we find that AGN hosts are a factor $\sim 2$ smaller in FIR size than the comparison galaxies. The difference reaches up to the $5.8 \sigma$ level, for the bin centered on $\log \left(L_{\mathrm{FIR}}\right.$ $\left.\left[L_{\odot}\right]\right)=9.75$. No such difference is seen for higher FIR luminosity (higher SFR), but the statistics for the AGN hosts is more limited in this regime. This is even more true for the PG QSOs studied by Lutz et al. (2016). The size of their FIR emission (their Fig. 10 right) is fully consistent with that of the BAT hosts at same FIR luminosity in Fig. 2. The PG QSO statistics are too limited for any meaningful separation into subsamples grouped by FIR luminosity, but the general pattern with smallish sizes for the very few modest FIR luminosity PG hosts and the sizes close to comparison galaxies for the more numerous higher $L_{\mathrm{FIR}}$ is consistent with what is reported here with better statistics for the BAT hosts.

These results are consistent with the important $\lesssim 2 \mathrm{kpc}$ point source contributions reported for PACS far-infrared images of

OT2_jrigby_3, OT2_jsmith01_2, OT2_kwestfal_2, OT2_lhunt_4, OT2_mboquien_3, OT2_mboquien_4, OT2_mhaynes_2. 

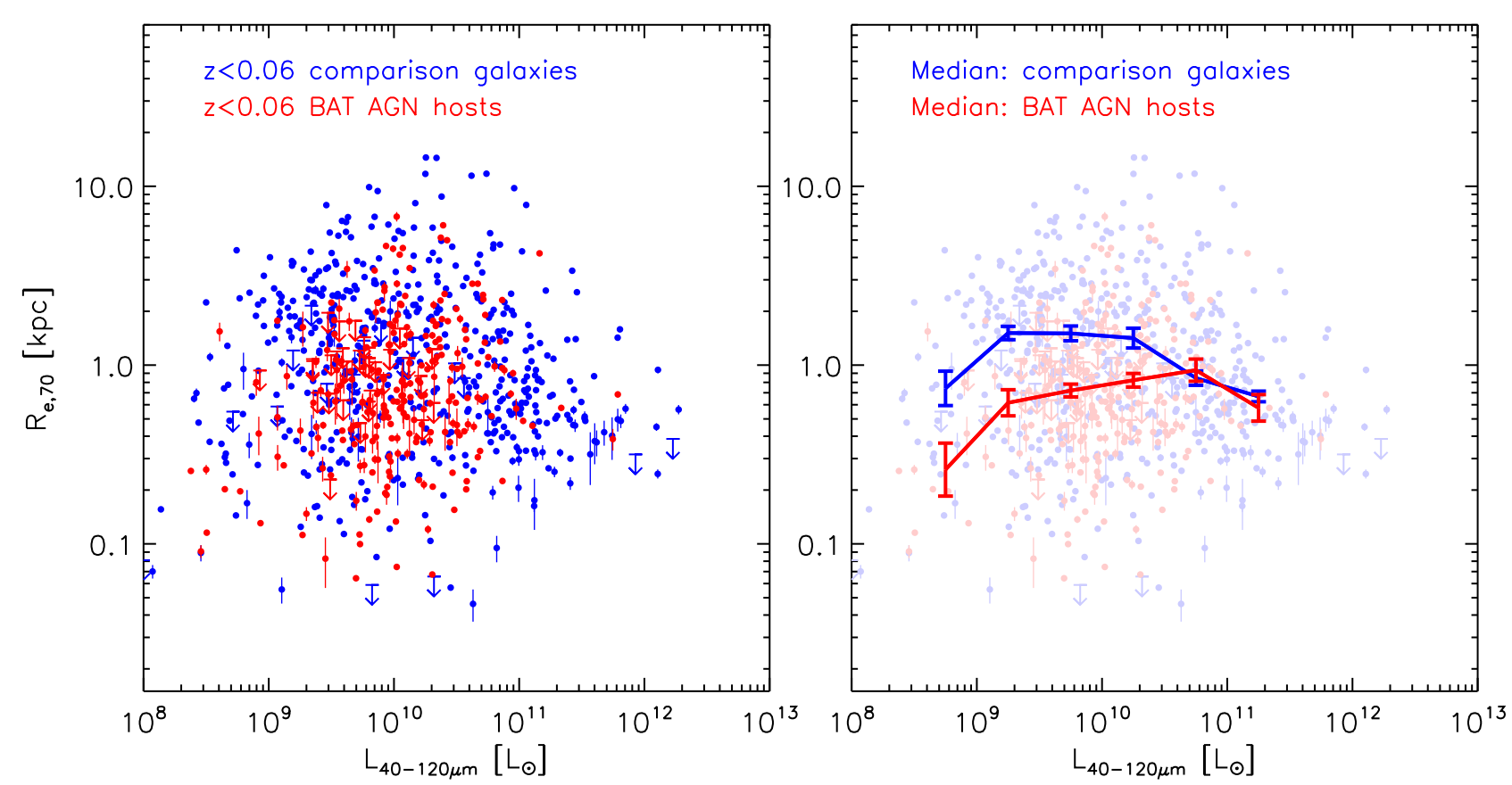

Fig. 2. Far-infrared $70 \mu \mathrm{m}$ half light radii as a function of FIR luminosity, for the Herschel-BAT sample and the comparison sample. Left panel: as observed. Right panel: median values and the $1 \sigma$ uncertainties of these medians are overplotted for 0.5 dex bins in $L_{\mathrm{FIR}}$.

Table 1. FIR half light radii of BAT AGN hosts and comparison galaxies.

\begin{tabular}{crrrrrrrr}
\hline \hline Bin center & \multicolumn{3}{c}{ BAT AGN hosts } & \multicolumn{4}{c}{ Comparison galaxies } \\
$\log \left(L_{\mathrm{FIR}}\left[L_{\odot}\right]\right)$ & $N$ & \multicolumn{2}{c}{$\log \left(R_{\mathrm{e}, 70}[\mathrm{kpc}]\right)$} & & $N$ & \multicolumn{2}{c}{$\log \left(R_{\mathrm{e}, 70}[\mathrm{kpc}]\right)$} \\
& & Median & \multicolumn{1}{l}{ MAD } & $\sigma$ & & Median & MAD & $\sigma$ \\
\hline 8.75 & 9 & -0.585 & 0.300 & 0.148 & 29 & -0.130 & 0.349 & 0.096 \\
9.25 & 29 & -0.211 & 0.265 & 0.073 & 94 & 0.179 & 0.244 & 0.037 \\
9.75 & 99 & -0.142 & 0.240 & 0.036 & 111 & 0.177 & 0.294 & 0.041 \\
10.25 & 93 & -0.085 & 0.248 & 0.038 & 92 & 0.151 & 0.357 & 0.055 \\
10.75 & 38 & -0.028 & 0.257 & 0.062 & 96 & -0.073 & 0.265 & 0.040 \\
11.25 & 4 & -0.239 & 0.100 & 0.074 & 62 & -0.175 & 0.158 & 0.030 \\
\hline
\end{tabular}

Notes. MAD $=$ median absolute deviation. Uncertainty $\sigma=1.4826 \times \mathrm{MAD} / \sqrt{N}$.

the BAT sample by Mushotzky et al. (2014), and extend that work via improved size measurements and a systematic comparison to non-BAT galaxies.

To assist the discussion of the size difference between AGN hosts and comparison galaxies, we define for each BAT AGN host in the range $8.5 \leq \log \left(L_{\mathrm{FIR}}\left[L_{\odot}\right]\right) \leq 10.5$ a "size excess" quantity

$\Delta R_{\mathrm{e}}=\log \left(R_{\mathrm{e}, 70, \mathrm{AGN}}\right)-\log \left(\operatorname{Median}\left(R_{\mathrm{e}, 70, \text { Comparison }}\right)\right)$,

where the median is taken over all comparison galaxies in the 0.5 dex wide $L_{\mathrm{FIR}}$ bin of the given AGN host. By referencing AGN to the value in their bin, we consider the minor trends for the comparison galaxies seen in Fig. 2, but are able to keep the full $8.5 \leq \log \left(L_{\text {FIR }}\left[L_{\odot}\right]\right) \leq 10.5$ statistics.

\section{Discussion}

\subsection{Compact star formation or AGN heated dust?}

Two hypotheses can clearly explain the factor of $\sim 2$ smaller sizes of the FIR emission in moderately FIR luminous AGN hosts.
First, a more compact gas distribution would lead to more compact star formation and FIR emission and, at least in the longterm average, boost feeding of the central SMBH, making the galaxy more likely to be detected as an AGN. Second, direct heating of dust by the AGN radiation could lead to a central emission spike that is significant not only for the mid-infrared AGN emission from warm dust, but even out to the far-infrared. This second effect should be more prominent for the most powerful AGN and for those with the highest ratio of $L_{\mathrm{bol}, \mathrm{AGN}}$ and $L_{\mathrm{FIR}}$.

In Fig. 3, we plot the size excess $\Delta_{\text {Re }}$ (Eq. (1)) for the BAT AGN as a function of AGN bolometric luminosity, and as a function of the ratio of AGN bolometric luminosity and FIR luminosity. No clear trend is seen in either diagram, and compact FIR emission ( small $\Delta R_{\mathrm{e}}$ ) is also observed for BAT systems with small $L_{\mathrm{bol}, \mathrm{AGN}}$ and low ratio $L_{\mathrm{bol}, \mathrm{AGN}} / L_{\mathrm{FIR}}$.

The lower panel of Fig. 3 can also be directly compared to expectations for the "intrinsic" infrared SED of AGN, i.e., the SED of dust that is directly heated by the AGN, excluding the stellar heated host contribution. These intrinsic SEDs are very difficult to model from first principles if the considerable freedom to modify the geometric arrangement of dust around the AGN is taken into consideration. It is worth noting, however, that 

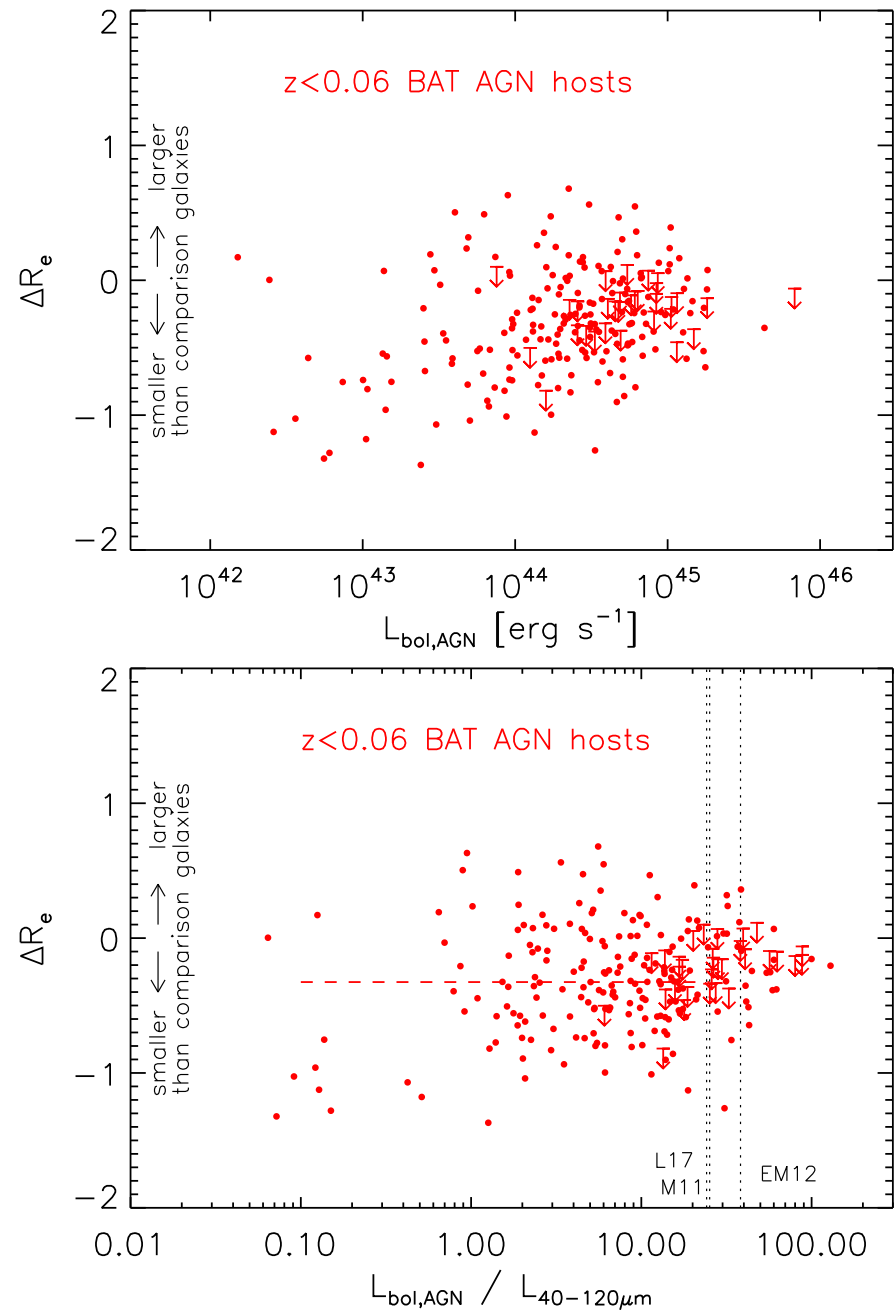

Fig. 3. "Size excess" $\Delta R_{\mathrm{e}}$ (Eq. (1)) of BAT AGN hosts compared to the median value for comparison galaxies. Top: as a function of AGN bolometric luminosity. Bottom: as a function of the ratio of AGN bolometric luminosity to far-infrared luminosity. Vertical dotted lines mark $L_{\mathrm{bol}} / L_{\mathrm{FIR}}$ for three versions of pure AGN "intrinsic" SEDs: the extended Mor \& Netzer (2012) SED, an SED derived by Lani et al. (2017), and a Mullaney et al. (2011) "Hi. Lum." SED, extended from $6 \mu \mathrm{m}$ down to $1 \mu \mathrm{m}$. A size deficit for the AGN hosts is also observed for $L_{\mathrm{bol}, \mathrm{AGN}} / L_{\mathrm{FIR}}<25$ (dashed horizontal median line), where the AGN contribution to the FIR should be minor according to these SEDs.

for radiative transfer models with plausible geometric assumptions (Schneider et al. 2015; Duras et al. 2017), AGN heated emission falls short of fully dominating the far-infrared even for some of the most luminous high-z QSOs. Empirical determinations of the intrinsic SED, by subtraction of a host SED from the observed total SED, are also uncertain. Variations among such empirical SEDs arise from the uncertainty in measuring the host SFR (e.g., via the mid-infrared PAH emission), and in adopting the appropriate host SED template for the given SFR. We overplot the expectations for a number of intrinsic SED templates from the literature in the lower panel of Fig. 3. These intrinsic SEDs serve to indicate a range of recent attempts to determine intrinsic AGN SEDs in the infrared (see also Netzer et al. 2007; Lyu \& Rieke 2017). We assume for these $L_{\mathrm{bol}, \mathrm{AGN}}=2 \times L_{\text {Torus }}$, where $L_{\text {Torus }}$ is taken as the $1-1000 \mu \mathrm{m}$ integral over the intrinsic AGN SED. Mor \& Netzer (2012) derive an intrinsic SED with relatively low FIR emission, which Netzer et al. (2014) extrapolated to longer wavelengths by a modified blackbody. The intrinsic SED of Mullaney et al. (2011; their "Hi. Lum" SED which is appropriate for our $L_{\mathrm{bol}, \mathrm{AGN}}$ range, and which we have amended with the Mor \& Netzer 2012, SED for 1-6 $\mu \mathrm{m}$ ) and the recent PAH-based SED of Lani et al. (2017) have stronger FIR emission. The even stronger FIR suggested by Symeonidis et al. (2016) is problematic (Lyu \& Rieke 2017; Lani et al. 2017) and is not used here. The vertical dotted lines in Fig. 3 are based on the simple $40-120 \mu \mathrm{m}$ integral which is about twice $v L_{v, 70}$, with variation between intrinsic AGN SEDs. They are hence conservatively overestimating the AGN effect at the PACS wavelengths proper.

If one of these intrinsic AGN SEDs were universal and the small FIR sizes of BAT hosts were due to AGN heated dust, a downward trend should be expected to the right of the corresponding line in Fig. 3 bottom. This is not observed. Some effect might still be hidden behind limited statistics at very high $L_{\mathrm{bol}, \mathrm{AGN}} / L_{\mathrm{FIR}}$ and the fact that there is no strictly universal AGN SED, as also clearly seen from slope variations in the midinfrared (e.g., Netzer et al. 2007; Lyu et al. 2017). Still, our results may be slightly in favor of the more FIR-weak intrinsic SEDs (rightmost vertical line). More importantly, the median $\Delta_{\mathrm{Re}}$ is -0.33 for the AGN hosts with $L_{\mathrm{bol}, \mathrm{AGN}} / L_{\mathrm{FIR}}<25$. which means that we find the same factor of $\sim 2$ size deficit for the AGN that are weak compared to their host, and where AGN heated FIR dust should provide an unimportant contribution given the intrinsic AGN SEDs.

We conclude that the FIR size deficit of BAT AGN hosts is mostly a host property, reflecting a different distribution of gas and star formation than in comparison galaxies of same $L_{\mathrm{FIR}}$. A dominant AGN contribution to the FIR emission and its compactness may nevertheless be present in some individual BAT sources. This may also be true for some very FIR-faint BAT AGN that do not enter our analysis because of insufficient $70 \mu \mathrm{m} \mathrm{S} / \mathrm{N}$.

\subsection{Circumnuclear vs. disk scale star formation in AGN hosts}

We have established a tendency for star formation in BAT AGN hosts, as traced by far-infrared emission, to be spread over a region that is typically only half the size of that in comparison galaxies. Turned around, this implies that for a given star formation rate, or related to this a given molecular gas mass, an AGN is more likely to be fuelled if that supply is more centrally concentrated. This is completely plausible in an AGN-host coevolution where black hole and star formation are fed from a common reservoir.

The large scatter in FIR sizes for both AGN hosts and comparisons and the need for a large sample to establish this size difference are obvious consequences of the diverse star formation morphologies of local galaxies and of the different spatial scales of star formation and black hole accretion. Their link, while present, is not tight. For the same reason, we do not see a relation to AGN bolometric luminosity over the range of the BAT sample (Fig. 3); accretion may vary over such a dynamic range much more rapidly than SF phenomena do.

While more concentrated than in non-active galaxies, the star formation in the BAT AGN hosts typically does not occur in extreme events. With typical star formation rates of $\sim 1 M_{\odot} \mathrm{yr}^{-1}$ and half light radii of $\lesssim 1 \mathrm{kpc}$, they correspond to a fairly undramatic local disk galaxy SFR that just is somewhat more spatially concentrated. Very long periods would be needed to significantly modify the stellar population over this spatial scale. 
At this point, it is interesting to return to the fact that we observe a far-infrared size deficit for AGN hosts only up to modest $L_{\mathrm{FIR}}$, or equivalently $S F R \lesssim 6 M_{\odot} \mathrm{yr}^{-1}$ (Fig. 2). No significant size difference is observed for higher FIR luminosity. With the more limited statistics in these bins, a factor of $\sim 2$ size difference (as observed for the lower $L_{\mathrm{FIR}}$ bins) is excluded at the $4.7 \sigma$ level for the bin centered on $\log \left(L_{\mathrm{FIR}}\right)=10.75$ and $2.8 \sigma$ for the bin centered at $\log \left(L_{\mathrm{FIR}}\right)=11.25$. While even larger samples would be desirable to ultimately establish this different behavior, we can already speculate that we are starting to see a consequence of local IR-luminous galaxies often not having star formation spread over a large disk. If reaching high IR luminosities already requires compressing the limited gas content of local galaxies, for example by interactions or mergers, then favorable conditions for high BHAR will be granted automatically as a side effect. This agrees with the well-known frequent presence of AGN in IR luminous galaxies (e.g., Lutz et al. 1998; Veilleux et al. 1995, 1999, 2009).

\section{Conclusions}

We have analyzed the half light radius of far-infrared $70 \mu \mathrm{m}$ emission $R_{\mathrm{e}, 70}$ in the hosts of $277 z<0.06$ Swift-BAT selected AGN and $515 z<0.06$ non-BAT comparison galaxies, using two-dimensional Gaussian fits and subtraction of the PSF width in quadrature. We find the following:

- For both AGN and comparison galaxies, there is a large ( $\sim 2$ dex) size scatter, reflecting the wide range of star formation distributions in local galaxies.

- At modest $\log \left(L_{\mathrm{FIR}}\left[L_{\odot}\right]\right) 8.5-10.5\left(S F R \lesssim 6 M_{\odot} \mathrm{yr}^{-1}\right)$, the median FIR size of AGN hosts is $R_{\mathrm{e}, 70} \lesssim 1 \mathrm{kpc}$, a factor of about 2 smaller than the non-BAT comparisons. No such difference is observed at higher far-infrared luminosities.

- This size deficit is mostly caused by a more compact distribution of star formation and gas in the AGN hosts, but a contribution of compact AGN-heated dust cannot be excluded for some objects with extreme $L_{\mathrm{bol}, \mathrm{AGN}} / L_{\mathrm{FIR}}$.

- In the context of AGN-host coevolution where SFR and BHAR are fed from the same general gas supply, these findings argue for a more compact SFR and gas distribution favoring AGN feeding. Large scatter remains in this link because of the large $\sim \mathrm{kpc}$ scale probed by the SFR data and because of the possibility of rapid accretion variations that smear the distinction of AGN and comparison galaxies.

- The lack of a size difference between AGN hosts and comparison galaxies at higher $L_{\mathrm{FIR}}$ may relate to such infrared luminosities mostly requiring compact star formation.

Acknowledgements. We thank the referee for the comments that helped improve the paper. PACS has been developed by a consortium of institutes led by MPE (Germany) and including UVIE (Austria); KUL, CSL, IMEC (Belgium); CEA, OAMP (France); MPIA (Germany); IFSI, OAP/OAT, OAA/CAISMI, LENS, SISSA (Italy); IAC (Spain). This development has been supported by the funding agencies BMVIT (Austria), ESA-PRODEX (Belgium), CEA/CNES (France), DLR (Germany), ASI (Italy), and CICYT/MCYT (Spain). This work has made use of the NASA/IPAC Extragalactic Database (NED), which is operated by the Jet Propulsion Laboratory, California Institute of Technology, under contract with the National Aeronautics and Space Administration. This research has made use of the NASA/IPAC Infrared Science Archive, which is operated by the Jet Propulsion Laboratory, California Institute of Technology, under contract with the National Aeronautics and Space Administration.

\section{References}

Baumgartner, W. H., Tueller, J., Markwardt, C. B., et al. 2013, ApJS, 207, 19 Boselli, A., Eales, S., Cortese, L., et al. 2010, PASP, 122, 261

Chabrier, G. 2003, PASP, 115, 763

Chen, C.-T. J., Hickox, R. C., Alberts, S., et al. 2013, ApJ, 773, 3

Cortese, L., Fritz, J., Bianchi, S., et al. 2014, MNRAS, 440, 942

Delvecchio, I., Lutz, D., Berta, S., et al. 2015, MNRAS, 449, 373

Diamond-Stanic, A. M., \& Rieke, G. H. 2012, ApJ, 746, 168

Duras, F., Bongiorno, A., Piconcelli, E., et al. 2017, A\&A, 604, A67

García-Burillo, S., Combes, F., Hunt, L. K., et al. 2003, A\&A, 407, 485

García-Burillo, S., Combes, F., Schinnerer, E., Boone, F., \& Hunt, L. K. 2005, A\&A, 441, 1011

García-González, J., Alonso-Herrero, A., Hernán-Caballero, A., et al. 2016, MNRAS, 458, 4512

Graciá-Carpio, J., Wetzstein, M., \& Roussel, H. 2015, ArXiv e-prints [arXiv: 1512.03252]

Haan, S., Schinnerer, E., Emsellem, E., et al. 2009, ApJ, 692, 1623

Kauffmann, G., Heckman, T. M., Tremonti, C., et al. 2003, MNRAS, 346, 1055 Kennicutt Jr., R. C. 1998, ARA\&A, 36, 189

Kennicutt, R. C., Calzetti, D., Aniano, G., et al. 2011, PASP, 123, 1347

Koss, M., Mushotzky, R., Veilleux, S., et al. 2011, ApJ, 739, 57

Lani, C., Netzer, H., \& Lutz, D. 2017, MNRAS, 471, 59

Lutz, D., Spoon, H. W. W., Rigopoulou, D., Moorwood, A. F. M., \& Genzel, R. 1998, ApJ, 505, L103

Lutz, D., Berta, S., Contursi, A., et al. 2016, A\&A, 591, A136

Lyu, J., \& Rieke, G. H. 2017, ApJ, 841, 76

Lyu, J., Rieke, G. H., \& Shi, Y. 2017, ApJ, 835, 257

Meléndez, M., Mushotzky, R. F., Shimizu, T. T., Barger, A. J., \& Cowie, L. L. 2014, ApJ, 794, 152

Mor, R., \& Netzer, H. 2012, MNRAS, 420, 526

Mullaney, J. R., Alexander, D. M., Goulding, A. D., \& Hickox, R. C. 2011, MNRAS, 414, 1082

Mullaney, J. R., Pannella, M., Daddi, E., et al. 2012, MNRAS, 419, 95

Mushotzky, R. F., Shimizu, T. T., Meléndez, M., \& Koss, M. 2014, ApJ, 781, L34

Netzer, H., Lutz, D., Schweitzer, M., et al. 2007, ApJ, 666, 806

Netzer, H., Mor, R., Trakhtenbrot, B., Shemmer, O., \& Lira, P. 2014, ApJ, 791, 34

Pilbratt, G. L., Riedinger, J. R., Passvogel, T., et al. 2010, A\&A, 518, L1

Poglitsch, A., Waelkens, C., Geis, N., et al. 2010, A\&A, 518, L2

Rosario, D. J., Santini, P., Lutz, D., et al. 2012, A\&A, 545, A45

Sanders, D. B., Mazzarella, J. M., Kim, D.-C., Surace, J. A., \& Soifer, B. T. 2003, AJ, 126, 1607

Schneider, R., Bianchi, S., Valiante, R., Risaliti, G., \& Salvadori, S. 2015, A\&A, 579, A60

Shao, L., Lutz, D., Nordon, R., et al. 2010, A\&A, 518, L26

Shimizu, T. T., Mushotzky, R. F., Meléndez, M., Koss, M., \& Rosario, D. J. 2015, MNRAS, 452, 1841

Shimizu, T. T., Meléndez, M., Mushotzky, R. F., et al. 2016, MNRAS, 456, 3335 Shimizu, T. T., Mushotzky, R. F., Meléndez, M., et al. 2017, MNRAS, 466, 3161 Stanley, F., Harrison, C. M., Alexander, D. M., et al. 2015, MNRAS, 453, 591 Symeonidis, M., Giblin, B. M., Page, M. J., et al. 2016, MNRAS, 459, 257

Veilleux, S., Kim, D.-C., Sanders, D. B., Mazzarella, J. M., \& Soifer, B. T. 1995, ApJS, 98, 171

Veilleux, S., Kim, D.-C., \& Sanders, D. B. 1999, ApJ, 522, 113

Veilleux, S., Kim, D.-C., Rupke, D. S. N., et al. 2009, ApJ, 701, 587

Wen, X.-Q., Wu, H., Zhu, Y.-N., et al. 2013, MNRAS, 433, 2946

Wright, E. L., Eisenhardt, P. R. M., Mainzer, A. K., et al. 2010, AJ, 140, 1868 


\section{Appendix A: Far-infrared sizes for BAT sample and reference sample}

Table A.1 lists the derived $70 \mu \mathrm{m}$ sizes and other quantities for our samples. A portion is shown here for guidance, the full table is available at the CDS.

Table A.1. BAT and reference samples.

\begin{tabular}{|c|c|c|c|c|c|c|c|c|c|}
\hline $\begin{array}{l}\text { Name } \\
\text { (1) }\end{array}$ & $\begin{array}{r}\text { RA } \\
\mathrm{J} 2000 \\
(2) \\
\end{array}$ & $\begin{array}{r}\text { Dec } \\
\mathrm{J} 2000 \\
(3) \\
\end{array}$ & $\begin{array}{c}\text { Sample } \\
(4)\end{array}$ & $\begin{array}{r}\text { Scale } \\
\mathrm{kpc} /{ }^{\prime \prime} \\
(5) \\
\end{array}$ & & $\begin{array}{l}\text { e,70 } \\
\text { pc } \\
6) \\
\end{array}$ & $\begin{array}{l}\text { Band } \\
(7) \\
\end{array}$ & $\begin{array}{r}\log \left(L_{\mathrm{FIR}}\right) \\
L_{\odot} \\
(8) \\
\end{array}$ & $\begin{array}{r}\log \left(L_{14-195 \mathrm{keV}}\right) \\
\mathrm{erg} / \mathrm{s} \\
(9)\end{array}$ \\
\hline UGC 12914 & 0.4110 & 23.4825 & Ref & 0.297 & 5.876 & \pm 0.032 & 70 & 10.16 & \\
\hline UGC 12915 & 0.4256 & 23.4956 & Ref & 0.295 & 2.086 & \pm 0.003 & 70 & 10.49 & \\
\hline PG 0003+199 & 1.5805 & 20.2027 & BAT & 0.519 & 0.399 & \pm 0.075 & 70 & 9.77 & 43.45 \\
\hline NGC 23 & 2.4716 & 25.9237 & Ref & 0.310 & 1.004 & \pm 0.003 & 70 & 10.75 & \\
\hline NGC 34 & 2.7780 & -12.1076 & Ref & 0.398 & 0.253 & \pm 0.017 & 70 & 11.28 & \\
\hline Arp $256 \mathrm{~N}$ & 4.7081 & -10.3604 & Ref & 0.549 & 3.159 & \pm 0.016 & 70 & 10.34 & \\
\hline Arp $256 \mathrm{~S}$ & 4.7122 & -10.3771 & Ref & 0.544 & 0.876 & \pm 0.010 & 70 & 11.13 & \\
\hline 2MASX J00253292+6821 & 6.3847 & 68.3625 & BAT & 0.246 & 0.307 & \pm 0.050 & 70 & 9.07 & 42.77 \\
\hline CGCG 535-012 & 9.0875 & 45.6649 & BAT & 0.934 & 1.909 & \pm 0.287 & 70 & 10.03 & 43.92 \\
\hline
\end{tabular}

Notes. (1) Source name. (2), (3) Far-infrared source position, as measured from the Gaussian fits. (4) Sample - BAT or reference (Ref). (5) Scale for the adopted distance and cosmology. (6) Far-infrared half light radii at $70 \mu \mathrm{m}$. Half light radii are based on subtracting in quadrature observed width and PSF width. The errors are only statistical and do not include systematics due to the non-Gaussianity of the real source structure and the PSF. (7) Band used to derive $R_{\mathrm{e}, 70} \cdot R_{\mathrm{e}, 70}$ is derived from Herschel-PACS $70 \mu \mathrm{m}$ data, with few exceptions where it was scaled from $R_{\mathrm{e}, 100}$ (measured at $100 \mu \mathrm{m}$ ), using $R_{\mathrm{e}, 70}=0.85 \times R_{\mathrm{e}, 100}$. These are marked 100 in the Band column. (8) $40-120 \mu \mathrm{m}$ far-infrared luminosity. (9) Swift-BAT extremely hard X-ray luminosity. 